\title{
VSN16R - a novel treatment for spasticity in experimental multiple sclerosis
}

\section{Thomas E. Williams ${ }^{1}$, Gareth Pryce ${ }^{1}$, Gavin Giovannoni, David L Selwood ${ }^{2}$, David Baker ${ }^{1}$}

Neuroinflammation Group, Blizard Institute Bart's and the London school of Medicine \& Dentistry, Queen Mary University of London, ${ }^{2}$ UCL Wolfson Institute for Biomedical Research, University

College London, UK

Current symptomatic treatments for spasticity in multiple sclerosis often exhibit intolerable sideeffects that limit their use. We synthesized a novel compound, VSN16R, which exhibited anti-spastic activity in experimental autoimmune encephalomyelitis (EAE) in mice and was as active as baclofen and cannabinoids but lacked their sedative side-effects. The drug was found to be a novel, potent BKCa calcium activated potassium channel modulator in vitro. VSN16R was orally active and remarkably well-tolerated, with over a thousand fold therapeutic window, and demonstrated no obvious adverse neurobehavioural effects in mice. It was also well tolerated in other larger animal species and importantly in humans, where phase 1 studies found VSN16R to produced high oral bioavailability and no serious adverse behavioural or physiological events at supra-therapeutic plasma concentrations. VSN16R has the potential to inhibit pathogenic spinal cord hyperreflexia and can drive neuronal potassium-induced hyperpolarisation to limit spasticity via BKCa opening. This study identifies a novel target for control of spasticity and suggests that VSN16R may be a useful novel therapeutic, which offers tolerability advantages over existing treatments. This may facilitate adoption of earlier treatment following to development of spasticity in MS. A phase II clinical trial (NCT02542787) is currently in progress.

Disclosures: Williams has no disclosures. Baker, Pryce, Giovannoni and Selwood are shareholders in Canbex therapeutics Ltd., a university spin-out company developing VSN16 